\title{
Nucleation by Crystalline Particles
}

Cite as: J. Chem. Phys. 38, 237 (1963); https://doi.org/10.1063/1.1733468

Submitted: 30 July 1962 . Published Online: 20 July 2004

\section{N. H. Fletcher}

\section{ARTICLES YOU MAY BE INTERESTED IN}

Size Effect in Heterogeneous Nucleation

The Journal of Chemical Physics 29, 572 (1958); https://doi.org/10.1063/1.1744540

Kinetics of Heterogeneous Nucleation

The Journal of Chemical Physics 18, 198 (1950); https://doi.org/10.1063/1.1747588

Erratum: Size Effect in Heterogeneous Nucleation

The Journal of Chemical Physics 31, 1136 (1959); https://doi.org/10.1063/1.1730526

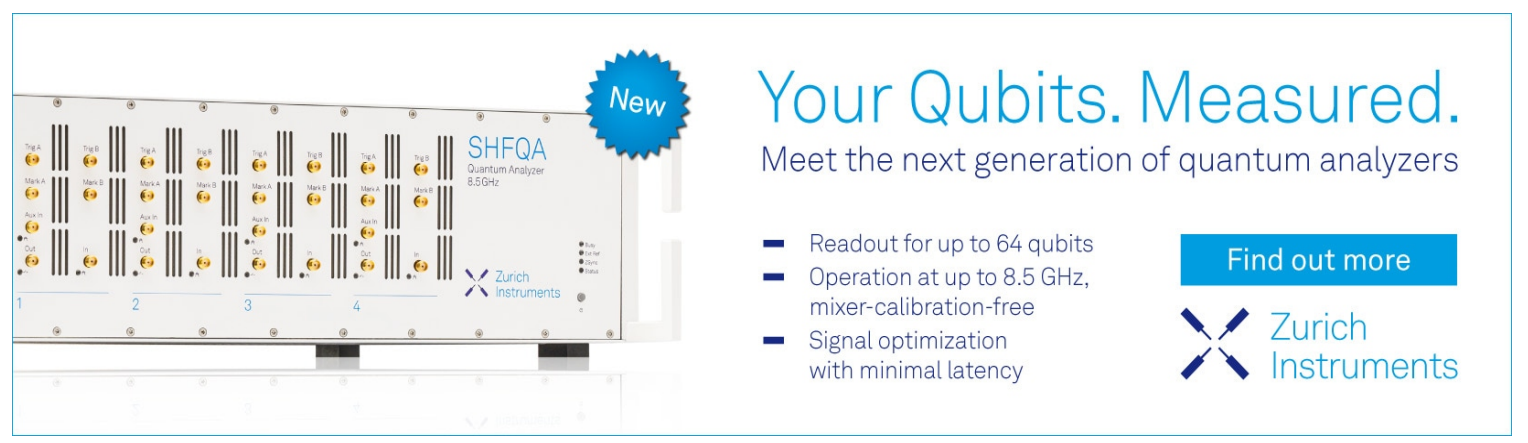




\title{
Nucleation by Crystalline Particles*
}

\author{
N. H. FLETCHER \\ Department of Physics, University of New England, Armidale, N.S.W., Ausiralia
}

(Received 30 July 1962)

\begin{abstract}
Classical nucleation theory is applied to the production of particles of a crystalline phase by growth upon small foreign particles. Various possible particle shapes are considered and general curves are derived which can be simply applied to a large variety of physical situations.
\end{abstract}

\section{INTRODUCTION}

$\mathbf{T}$ THE heterogeneous nucleation of a new phase by foreign particles suspended in the parent phase is of practical importance in many fields. Particularly important is an understanding of the way in which the efficiency of these foreign nuclei depends upon their size, shape, and surface properties.

In an earlier discussion of this problem, ${ }^{1}$ the present author considered the simple case in which both the nucleating particle and the embryo of the new phase growing upon it were spherical in shape. The results of this treatment have proved useful in understanding the behavior of aerosols of silver iodide in the nucleation of ice crystals in the atmosphere, ${ }^{2}$ as well as having application to other systems.

It is clear, however, that many nucleating particles cannot be regarded as even approximately spherical, and, in cases where the embryo of the new phase is crystalline, it too may depart significantly from the spherical approximation. It will be our purpose then, in the present paper, to consider the nucleation behavior of particles ranging in shape from disks to needles, and to take some account of the crystalline nature of the new phase. On the basis of results for such extreme cases it should be possible, by interpolation, to arrive at semiquantitative conclusions for nuclei and embryos of intermediate habits.

Whilst it is simplest to arrive at results for a particular substance whose physical properties are known explicitly, it is possible, with little sacrifice, to derive general results in terms of parameters which differ from one substance to another. Our discussion is therefore quite general, within the range of validity of certain assumptions which are later made explicit.

\section{CLASSICAL NUCLEATION THEORY}

Classical nucleation theory, as developed by Volmer and others, ${ }^{3}$ applies statistical arguments to the

\footnotetext{
* This research was supported by the Atmospheric Science Program, National Science Foundation, under NSF grant G19677.

${ }_{1}$ N. H. Fletcher, J. Chem. Phys. 29, 572 (1958); 31, 1136 (1959).

${ }_{2}^{2}$ N. H. Fletcher, J. Meteorology 16, 173, 249, 385 (1959), Discussions Faraday Soc. 30, 39 (1960).

${ }_{3}$ M. Volmer, Kinetik der Phasenbildung (Thedor Steinkopff Verlag, Dresden, 1939).
}

probability of growth of an embryo of a new phase from a metastable environment. The rate $J$ of nucleation of isolated elements of the new phase is given by an expression of the form

$$
J=K \exp \left(-\Delta G^{*} / k T\right),
$$

where $\Delta G^{*}$ is the free energy required for the formation of a single embryo of such a size that it is in unstable equilibrium with its environment, and $K$ is a constant which is determined by the kinetics of the situation.

$K$ can usually be determined, in order of magnitude, by relatively simple arguments, ${ }^{3}$ though many refinements are possible. Most interest, and most uncertainty, centers on the evaluation of $\Delta G^{*}$. It is usual here to apply macroscopic concepts like surface free energy rather freely to discussions of molecular groupings of quite small size. This procedure is of very marginal validity in many cases, but it is still used because the results obtained by such methods agree quite well with experiment in cases where they have been checked, $^{3,4}$ and because a more realistic molecular approach seems at present prohibitively difficult.

We consider, then, an embryo of the new phase 2 growing on a nucleating particle 3 within the parent phase 1 . If $V_{2}$ is the volume of the embryo and $S_{i j}$ is the area of the $i j$ interface, then the free energy involved in forming this embryo is

$$
\Delta G=\Delta G_{v} V_{2}+\sigma_{12} S_{12}+\left(\sigma_{23}-\sigma_{13}\right) S_{23},
$$

where $\Delta G_{v}$ is the free energy required to form unit volume of phase 2 from phase 1 and $\sigma_{i j}$ is the free energy per unit area of the $i j$ interface. It proves convenient to introduce the parameter $m$, defined by

$$
m \equiv\left(\sigma_{13}-\sigma_{23}\right) / \sigma_{12},
$$

to express the degree of compatibility between nucleus and embryo. It turns out that

$$
-1 \leq m \leq 1,
$$

with values of $m$ approaching unity indicating a high degree of compatibility, analogous to "wetting" in the case of a liquid embryo.

Once the geometry has been made explicit so that $V_{2}$

${ }^{4}$ S. Twomey, J. Chem. Phys. 30, 941 (1959). 
and the $S_{i j}$ are known, the maximum value of $\Delta G$ as a function of embryo size can be determined, and this is the quantity $\Delta G^{*}$ involved in Eq. (1).

The results of this procedure can be expressed in quite general form. Let $R$ be some characteristic dimension of the nucleus, and $r^{*}$ some characteristic dimension of the critical embryo which is in unstable equilibrium with its environment. Then if we define

we can write

$$
x \equiv R / r^{*},
$$

$$
\Delta G^{*}=\Delta G_{0}^{*} f(m, x),
$$

where $\Delta G_{0}{ }^{*}$ is the critical free energy for homogeneous nucleation in the absence of any foreign particle and $f(m, x)$ is a function whose form depends in detail upon the geometry of the situation.

For a spherical embryo

$$
\Delta G_{0} *=16 \pi \sigma_{12}^{3} / 3\left(\Delta G_{v}\right)^{2},
$$

while for an $n$-sided prismatic embryo, ${ }^{5}$ assuming $\sigma_{12}$ to be the same for all faces,

where

$$
\Delta G_{0}^{*}=8 \pi \sigma_{12}^{3} \xi /\left(\Delta G_{v}\right)^{2},
$$

$$
\xi=(n / \pi) \tan (\pi / n) .
$$

All this analysis neglects, to a large extent, the essential nature of a crystalline embryo. Thus, the dimensions of the embryo are considered to be continuously variable, and the two-dimensional nucleation of new molecular layers is neglected. In most cases this difficulty may be side-stepped by supposing the nucleus to contain a screw dislocation of small Burgers' vector, so that the embryo is similarly dislocated and two-dimensional nucleation is not required for growth. Detailed consideration of this point is complicated ${ }^{6}$ but it need not be discussed here. In the case of icecrystal nucleation a possible anomalous surface structure $^{7}$ may obviate the necessity for surface nucleation at the ice-vapor interface.

We assume in what follows that some such mechanism intervenes so that the expressions (6)-(9) are valid and the problem is essentially reduced to the geometrical one of evaluating $f(m, x)$.

\section{GEOMETRICAL CONSIDERATIONS}

Evaluation of the factor $f(m, x)$ for various geometries is straightforward though rather tedious. The methods involved have been described before. ${ }^{1,5}$ In the cases detailed below, the nucleus and embryo are considered either to be spherical, or to have the form of a circular cylinder. Various combinations of these forms are able to approximate most important nucleation situations. The neglect of other crystal faces does not have a large effect on the results and, in the case

\footnotetext{
${ }^{5}$ N. H. Fletcher, Australian J. Phys. 13, 408 (1960)

${ }^{6}$ See reference 5 , but note that the conclusion expressed in Sec. $\mathrm{V}$ is incorrect.

${ }^{7}$ N. H. Fletcher, Phil. Mag. 7, 255 (1962).
}

of the embryo, the rounding-off of corners is physically justified by entropy considerations. ${ }^{8}$

\section{Sphere on Sphere}

This case was treated in an earlier publication, ${ }^{1}$ and is included here for completeness. The geometrical factor $f(m, x)$ is here given by ${ }^{9}$

$$
\begin{aligned}
f(m, x) & =\frac{1}{2}+\frac{1}{2}[(1-m x) / g]^{3} \\
& +\frac{1}{2} x^{3}\left\{2-3[(x-m) / g]+[(x-m) / g]^{3}\right\} \\
& +\frac{3}{2} m x^{2}\{[(x-m) / g]-1\},
\end{aligned}
$$

where

$$
g=\left(1+x^{2}-2 m x\right)^{\frac{1}{5}} \text {. }
$$

In this and the other cases we consider, both $R$ and $r^{*}$ are unambiguously defined as radii, and $r^{*}$ is given by the relation

$$
r^{*}=-2 \sigma_{12} / \Delta G_{v}
$$

\section{Cylinder on Sphere}

In the case of a cylindrical embryo it is necessary to make some assumption about the relation between the free energies of different crystal faces. We here make the assumption that the free energies of base and cylinder faces are equal, so that the equilibrium habit of a small crystal is one in which the height equals the cylindrical diameter. Some such assumption is necessary if the calculation is to proceed, and this one appears to be reasonably realistic, extreme crystal habits not usually being the result of equilibrium processes.

For this case, then, we obtain the result

$$
\begin{array}{r}
f(m, x)=1-m x^{2}(1-h)-\frac{1}{2} x^{2}\left(1-h^{2}\right) \\
+\frac{1}{3} x^{3}(1-h)^{2}(2+h),
\end{array}
$$

where

$$
h=\left[\left(1-4 m x+4 x^{2}\right)^{\frac{1}{2}}-1\right] / 2 x .
$$

This result is only valid if

$$
h \geq\left(x^{2}-1\right)^{\frac{1}{2}} / x .
$$

If the condition (15) is not fulfilled, which only occurs for $x \geq 1$, then $f(m, x)$ is given by

$f(m, x)=\frac{1}{2}+\frac{2}{3} x^{3}-m x^{2}+\frac{1}{3}\left(x^{2}-1\right)^{\frac{1}{2}}\left(3 m x-2 x^{2}-1\right)$.

\section{Cylinder on Disk}

Nucleation upon a cylindrical nucleus is best considered through the two extreme cases in which the cylinder has the form either of a disk or of a needle.

\footnotetext{
${ }^{8}$ W. K. Burton, N. Cabrera, and F. C. Frank, Phil. Trans. Roy. Soc. (London) A243, 299 (1951).

${ }^{9}$ Note that $f(m, x)$ as defined here differs by a factor $\frac{1}{2}$ from that used in reference 1 .
} 
For the case of a disk we find, if $x \leq 1$,

$$
f(m, x)=1-\frac{1}{2} x(1+m),
$$

while, if $x \geq 1$,

$$
f(m, x)=(1-m) / 2 .
$$

\section{Cylinder on Cylinder}

As the other extreme we consider nucleation upon the curved surface of a long, needlelike cylinder. Nucleation upon the flat ends of the cylinder can be treated using results (17) and (18) above. For the present case we find

$f(m, x)=\pi^{-1}\left[\psi+x(x-2 m) \phi+\frac{1}{2} \sin 2 \psi-\frac{1}{2} x^{2} \sin 2 \phi\right]$,

where ${ }^{10}$

$$
\begin{gathered}
\phi=\cos ^{-1}[(x-m) / g], \\
\psi=\cos ^{-1}[(m x-1) / g],
\end{gathered}
$$

with

$$
0 \leq(\phi, \psi) \leq \pi
$$

and

$$
g=\left(1+x^{2}-2 m x\right)^{\frac{1}{2}}
$$

\section{RESULTS IN GENERAL FORM}

From the results of the preceding section and a knowledge of $K, \sigma_{12}$ and the form of $\Delta G_{v}$ for a given system, it is possible to calculate the nucleation rate $J$ as a function of supersaturation or supercooling. It is, however, more useful for our present purposes to make certain simplifying assumptions and approximations so that our results may be more generally applicable.

To this end we note that in many common nucleation situations, both in a vapor and a liquid environment, the kinetic constant in the rate equation (1) is of order of magnitude $10^{25} \mathrm{~cm}^{-2} \mathrm{sec}^{-1}$. We further note that a change of a few orders of magnitude in the value of this constant can be compensated for by a change of only a few percent in the value of $\Delta G_{v}$. Also, to a good approximation, the surface area of the nuclei we are considering is of order $10 R^{2}$. We may thus write ${ }^{11}$ for the constant $K$ in Eq. (1)

$$
K \sim 10^{26} R^{2},
$$

where $R$ is measured in $\mathrm{cm}$ and the rate $J$ is in nucleation events per nucleus per second.

We may now define an appreciable nucleation rate to be $J \sim 1 \mathrm{sec}^{-1}$, and, using Eqs. (1), (6), and (8) for a cylindrical embryo, we find that for an appreciable nucleation rate

$$
\Delta G_{v}^{2}=8 \pi \sigma^{3} f(m, x) / k T(60+2 \ln R),
$$

where we have set $\xi=1$ and have written $\sigma$ for $\sigma_{12}$.

\footnotetext{
${ }^{10} \phi$ and $\psi$ are the angles so designated in figure similar to figure 1 of reference 1 .

"I If $x<1$ it is better to write $r^{*}$ for $R$ in (24). We have done this in computing the curves shown later.
}

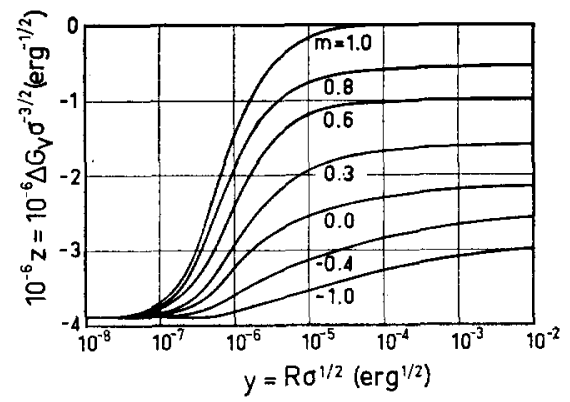

FIG. 1. General nucleation curves for the growth of an embryo of spherical habit upon a spherical nucleating particle. On each curve the nucleation rate is 1 particle ${ }^{-1} \sec ^{-1}, z$ is proportional to supersaturation or supercooling, as given by $(27)$, and $y$ to particle radius, according to (28). Parameter $m$ measures the compatibility of embryo and nucleus according to (3).

Using Eqs. (5) and (12), Eq. (25) can be put in the form

$$
\frac{\Delta G_{v}{ }^{2}}{\sigma^{3}}=\frac{8 \pi f\left(m,-\frac{1}{2} R \sigma^{\frac{1}{2}} \Delta G_{v} / \sigma^{3}\right)}{k T\left(60+2 \ln R \sigma^{\frac{1}{2}}-\ln \sigma\right)} .
$$

If we now recognize that for most systems with which we deal, $\sigma$ lies in the range $10-1000 \mathrm{erg} \mathrm{cm}^{-2}$, we can write $\ln \sigma \approx 5$. This done, we can introduce the quantities

$$
\begin{gathered}
z \equiv \Delta G_{v} / \sigma^{\frac{3}{2}}, \\
y \equiv R \sigma^{\frac{1}{2}}
\end{gathered}
$$

and, taking $T \approx 300^{\circ} \mathrm{K}$, we find

$$
z^{2} \approx \frac{3.0 \times 10^{14} f\left(m,-\frac{1}{2} y z\right)}{27+\ln y} \text {. }
$$

Similarly, for the case of a spherical embryo, using (7) instead of (8), we arrive at an equation differing from (29) only by the replacement of the factor $3.0 \times 10^{14}$ on the right side by the value $2.0 \times 10^{14}$.

While an analytic solution of (29), giving $z$ as a function of $y$ with $m$ a parameter, is not generally possible, it is quite simple to obtain a numerical solution by successive approximations. This has been done with the aid of a small computer, Sirius, and the results are shown in Figs. 1 to 4 .

The curves are, in all cases, qualitatively similar, but interesting quantitative differences are apparent. Geometrically matching cases such as sphere-onsphere or cylinder-on-cylinder, shown in Figs. 1 and 4, respectively, produce very similar curves, except that $\left|\Delta G_{v}\right|$ values are rather greater in the case of a cylindrical embryo. When the habits are not similar, however, as in the case of a cylinder growing on a sphere, the nucleation efficiency of quite large particles with $m$ values near unity is very markedly reduced. This can be clearly seen in Fig. 2. The case of a cylindrical embryo growing on a flat nucleus, as shown in Fig. 3, is interesting for the flatness of the curves for $x>1$ and the sharp decrease in nucleation efficiency for $x<1$. 


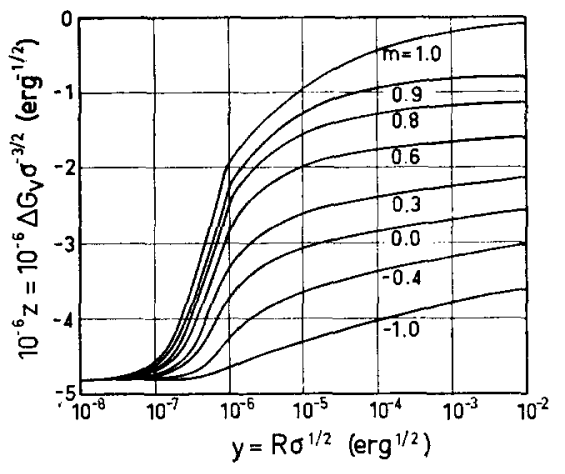

Fig. 2. General nucleation curves for the growth of an embryo of cylindrical habit upon a spherical particle.

\section{USE OF THE GENERAL CURVES}

As pointed out above, the curves given in Figs. 1 to 4 have been derived on the assumptions:

(i) that the interfacial free energy $\sigma_{12}$ lies in the range $10-1000$ erg $\mathrm{cm}^{-2}$,

(ii) that the kinetic constant $K$ in Eq. (1) is of order $10^{25} \mathrm{~cm}^{-2} \mathrm{sec}^{-1}$,

(iii) that an appreciable nucleation rate $J$ is of order 1 particle ${ }^{-1} \mathrm{sec}^{-1}$,

(iv) that the temperature is near $300^{\circ} \mathrm{K}$.

The curves should not be in error by more than about $10 \%$ on either axis for $\sigma_{12}$ values in the stated range, for values of $J$ or $K$ differing by about three orders of magnitude from those given, and for temperatures in the range $250^{\circ}$ to $350^{\circ} \mathrm{K}$. For larger temperature variations the approximate relations

$$
\begin{aligned}
& z(T)=(300 / T)^{\frac{1}{2}} z\left(300^{\circ}\right), \\
& y(T)=(T / 300)^{\frac{1}{2}} y\left(300^{\circ}\right),
\end{aligned}
$$

may be used as a guide to the results to be expected.

Providing these assumptions are reasonably valid, the general curves may be used to make predictions of nucleation behavior in a particular system by giving

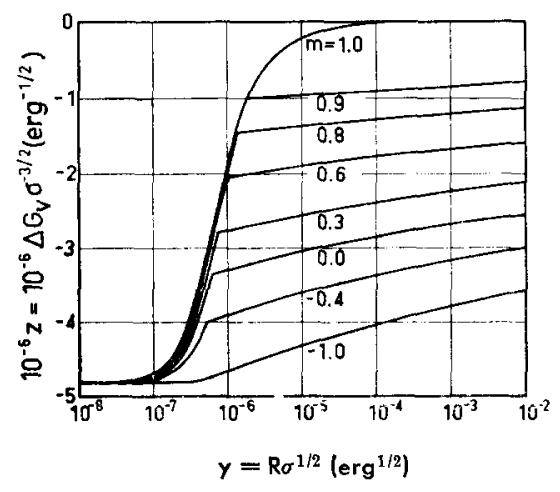

FIG. 3. General nucleation curves for the growth of an embryo of cylindrical habit upon a disk-shaped particle. explicit forms to the parameters $z$ and $y$ defined in Eqs. (27) and (28).

The driving free energy per unit volume $\Delta G_{v}$ can be expressed in the general form

$$
\Delta G_{v}=n\left(\mu_{2}-\mu_{1}\right)
$$

where $\mu_{i}$ is the chemical potential of molecules in phase $i$, and $n$ is the number of molecules per unit volume in the condensed phase 2. For condensation or sublimation from a supersaturated vapor of pressure $p$

$$
\Delta G_{v}=-n k T \ln \left(p / p_{0}\right),
$$

where $p_{0}$ is the vapor pressure in equilibrium with a plane surface of the condensed phase. Similarly for

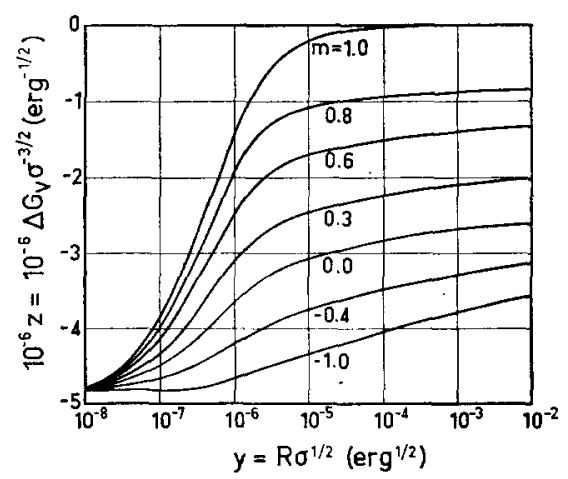

FIG. 4. General nucleation curves for the growth of an embryo of cylindrical habit upon the curved surface of a long needleshaped particle.

crystallization of a solute from a solution in which its activity is $a$,

$$
\Delta G_{v}=-n k T \ln \left(a / a_{0}\right) .
$$

The remaining case of interest is the freezing of a pure liquid, for which

$$
\Delta G_{v} \approx-\Delta S_{v} \Delta T
$$

where $\Delta S_{v}$ is the entropy of melting, per unit volume, and $\Delta T$ is the supercooling below the equilibrium freezing point.

\section{CONCLUSION}

We have derived general curves which may be used to make semiquantitative predictions about the behavior of small particles as heterogeneous nuclei for phase changes in various metastable systems.

The treatment is necessarily inexact because it is based upon the assumptions of classical nucleation theory, which neglects many molecular phenomena which may be of considerable importance. The success which has attended the application of even more simplified versions of this theory, however, allows one to hope that the present results will be a useful guide to average behavior in many common situations. 